\title{
BMJ Open Healthcare resource utilisation and cost associated with elevated potassium levels: a Danish population-based cohort study
}

\author{
Kun Kim, ${ }^{1}$ Reimar Wernich Thomsen, ${ }^{\circ} 2$ Sia Kromann Nicolaisen, ${ }^{2}$ \\ Lars Pål Hasvold, ${ }^{3}$ Eirini Palaka, ${ }^{4}$ Henrik Toft Sørensen ${ }^{2}$
}

To cite: Kim K, Thomsen RW, Nicolaisen SK, et al. Healthcare resource utilisation and cost associated with elevated potassium levels: a Danish population-based cohort study. BMJ Open 2019;9:e026465. doi:10.1136/ bmjopen-2018-026465

- Prepublication history and additional material for this paper are available online. To view these files, please visit the journal online (http://dx.doi. org/10.1136/bmjopen-2018026465).

Received 3 September 2018 Revised 8 February 2019 Accepted 15 February 2019

Check for updates

(C) Author(s) (or their employer(s)) 2019. Re-use permitted under CC BY-NC. No commercial re-use. See rights and permissions. Published by BMJ.

${ }^{1}$ Health Economics, AstraZeneca Nordic-Baltic, Södertälje, Sweden, Södertälje, Sweden ${ }^{2}$ Department of Clinical Epidemiology, Aarhus University Hospital, Aarhus N, Denmark

${ }^{3}$ Medical Department,

AstraZeneca Nordic, Oslo,

Norway

${ }^{4}$ Global Health Economics,

AstraZeneca, Cambridge,

Cambridge, UK

Correspondence to

Eirini Palaka;

eirini.palaka@astrazeneca.com

\section{ABSTRACT}

Objectives To investigate healthcare costs associated with hyperkalaemia (HK) among patients with chronic kidney disease (CKD), heart failure (HF) or diabetes. Design Before-after cohort study of patients with HK and matched patients without HK.

Setting Population-based databases covering primary and secondary care for the entire of Northern Denmark. Participants Patients with a first incident record of CKD $(n=78372), H F(n=14233)$ or diabetes $(n=37479)$ during 2005-2011. Among all patients experiencing a first HK event (potassium level $>5.0 \mathrm{mmol} / \mathrm{L}$ ), healthcare costs were compared during 6 months before and 6 months after the HK event. The same cost assessment was conducted 6 months before and after a matched index date in a comparison cohort of patients without HK. Primary and secondary outcome measures Mean costs of hospital care, general practice and dispensed drugs converted to 2018 Euros.

Results Overall, 17747 (23\%) CKD patients, 5141 (36\%) HF patients and $4183(11 \%)$ diabetes patients with a first HK event were identified. More than $40 \%$ of all HK patients across the patient groups had subsequent HK events with successively shorter times between the events. In CKD patients, overall mean costs were $€ 5518$ higher 6 months after versus before first HK, while $€ 441$ higher in matched CKD patients without HK, yielding HK-associated costs of $€ 5077$. Corresponding costs associated with a HK event were $€ 6018$ in HF patients, and $€ 4862$ in diabetes patients.

Conclusions Among CKD, HF and diabetes patients, an incident HK event was common, and a large proportion of the patients experienced recurrent HK events. Substantial increase in healthcare costs associated with a HK event was observed in the HK patients compared with non-HK patients. These results are important to better understand the potential economic impact of HK among high-risk comorbid patients in a real-wold setting and help inform decision-making for clinicians and healthcare providers.

\section{BACKGROUND}

Hyperkalaemia (HK) is defined as serum potassium levels above a reference range, usually $>5.0 \mathrm{mmol} / \mathrm{L}$, although more severe
Strengths and limitations of this study

- Access to laboratory test results from both primary and hospital care for the entire region's population of Northern Denmark (1 841902 residents, ie, 33\% of Denmark's population) linkable to nationwide data on morbidity, mortality and medication.

- Describing healthcare resource utilisation and corresponding costs in three main hyperkalaemia (HK) risk groups; patients with chronic kidney disease, heart failure or diabetes.

- Reported costs based on official Diagnosis-Related Groups and Danish Outpatient Grouping System charges.

- Any conclusions concerning causal mechanisms underlying HK outcomes and corresponding costs should be made with caution.

- Rather than precipitating a hospitalisation, elevated potassium levels may stem from an underlying condition that led to the hospitalisation.

potassium cut-off levels are often used in treatment decision guidelines $(\mathrm{eg},>5.5$ or $>6.0 \mathrm{mmol} / \mathrm{L}) .{ }^{12} \mathrm{HK}$ has been reported to occur in $3-8 \%$ of all hospitalised patients, independent of reason for hospitalisation, based on single clinic or health insurance-based cross-sectional studies. ${ }^{3-5}$ Patients older than 65 years with chronic kidney disease (CKD), heart failure (HF) diabetes or who use renin-angiotensin-aldosterone system inhibitors are at increased risk of experiencing HK. ${ }^{16-9}$ An elevated plasma potassium concentration may result in muscle weakness, paralysis, life-threatening effects on cardiac arrhythmias and sudden death. ${ }^{7}$ The latter two have been reported among patients with potassium levels $>6.0 \mathrm{mmol} / \mathrm{L} .^{10}{ }^{11}$ However, recent cohort analyses indicate that potassium levels $>5.0 \mathrm{mmol} / \mathrm{L}$ may also predict increased higher risk of cardiovascular events and short-term mortality among patients with 
an acute hospitalisation, as well as among patients with CKD or HF. ${ }^{12-17}$

HK has been associated with longer stay during acute hospitalisations, as well as an increased number of intensive care unit (ICU) stays and emergency department visits, primarily in the US healthcare system setting. ${ }^{117-19}$ However, the overall healthcare resource utilisation (HRU) and costs associated with HK have not been comprehensively studied in a full population-based setting outside the USA. To better understand the potential impact of new emerging drug therapies for HK, it is important to assess the true burden of HRU and costs in patients with HK events occurring in real-life, even if HK-related costs are unlikely to be causally driven by HK alone. The aims of this study were to investigate overall HRU and costs associated with HK in a real-world clinical setting in patients with an incident diagnosis of CKD, HF or diabetes.

\section{METHODS}

\section{Patient and public involvement}

This was a cohort study using local and nationwide register data and no patients were involved in the design of the study. The study design is based on previous experience on data that are of interest to healthcare providers.

\section{Data sources}

This cohort study was conducted in Northern Denmark, using routine laboratory test results from both primary and hospital care for the entire region's population (1 841902 residents, ie, 33\% of Denmark's population in June 2011). ${ }^{20}{ }^{21}$ Laboratory data were linked, via mandatory and unique civil personal registration numbers (assigned to each Danish resident), to hospital diagnoses and procedure data maintained in the Danish National Patient Registry (DNPR). The DNPR contains dates of hospital admission and discharge, emergency room visits and outpatient clinic visits as well as and procedures carried out in the hospital setting. ${ }^{22}$ Records of all drugs prescribed in an outpatient setting and dispensed from Danish pharmacies were obtained from the National Prescription Database and the Aarhus Prescription Database. ${ }^{21}{ }^{23}$ Data on general practitioner (GP) services were retrieved from the National Health Insurance Service Registry. ${ }^{24}$

\section{Study populations with CKD, HF or diabetes}

Overall, three disease cohorts with a first incident record of $\mathrm{CKD}$, $\mathrm{HF}$ or diabetes, respectively, were identified between 1 January 2005 and 30 June 2011, based on a combination of laboratory, drug prescription and hospital contact data. To ensure that our patients reflect true real-world disease cohorts with various possible comorbidities, an individual detected with more than one of these conditions (such as both incident diabetes and later incident CKD) during the study period could be included in more than one patient cohort, in each case starting on the detection date of the respective disease. Incident CKD was defined as the first occurrence of one of the following: (1) estimated glomerular filtration rate $(\mathrm{eGFR})<60 \mathrm{~mL} / \mathrm{min} / 1.73 \mathrm{~m}^{2}$ verified by at least two creatinine measurements $>90$ days apart, (2) an incident hospitalisation with a diagnosis of CKD or (3) hospital-based codes for renal dialysis. ${ }^{15}$ Incident HF was defined as the first occurrence of an inpatient hospital admission with a primary or secondary discharge diagnosis of $\mathrm{HF}^{25}$ Incident diabetes was defined as the first dispensed prescription for a glucose-lowering drug. ${ }^{26}$ To maximise the likelihood that the diseases were truly incident, we ensured that individuals had no previous record of the disease in question before study start (1 January 2005). Available look-back periods were back to 1977 for hospital codes (CKD or HF), to 1998 for prescription data (diabetes) and to 2000 for laboratory data (CKD).

\section{HK events}

Within each of the three cohorts, that is, individuals with a first record of CKD, $\mathrm{HF}$ and diabetes, respectively, we followed individuals for a first incident HK event occurring after being recorded for the first time with the respective disease. A HK event for a patient was identified as elevated blood potassium level $>5.0 \mathrm{mmol} / \mathrm{L}$ not preceded by a prior episode of elevated potassium within the previous month. ${ }^{152526}$ Thus, a patient with a first record of, for example, HF was eligible to have a first incident HK event as HF patient from the date of his or her first HF cohort entry, only if he or she had no previous HK event or if he or she had a previous HK event $>1$ month before the date of her first HF cohort entry. More severe HK events, $>5.5$ and $>6.0 \mathrm{mmol} / \mathrm{L}$, were detected the same way. The incidence of HK per 1000 person-years was calculated in the three cohorts. Following the first event, subsequent HK events were detected and reported, for potassium levels $>5.0,>5.5$ and $>6.0 \mathrm{mmol} / \mathrm{L}$, respectively. The incidences of subsequent HK events were presented per 1000 person-years within the median follow-up times between the events, and the healthcare setting where subsequent HK events were detected was reported.

\section{HK associated HRU and costs}

The overall self-controlled before-after analytic design is demonstrated in figure 1. Among patients who had experienced HK in each of the cohorts with CKD, HF or diabetes, we assessed the total number of hospital admissions (acute or planned non-acute inpatient hospitalisations, including dialysis procedures, ventilator treatment and ICU admissions), hospital outpatient visits, emergency room visits, GP contacts and drugs prescribed on an out-patient basis, during the periods 6 months before and 6 months after the incident HK event. The difference in overall HRU and resulting costs, during the 6 months before the HK event and 6 months after the HK event, was then calculated for each HK patient. If a HK patient was diagnosed with HK on 1 April 2008, for example, the total costs in the first 6 months after HK would be his or 
Patients with hyperkalemia

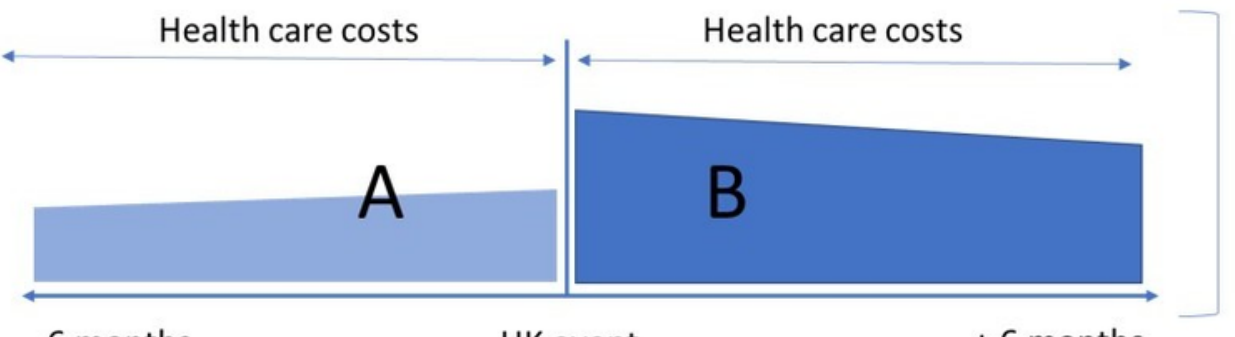

Costs $B-\operatorname{costs} A=$ Cost difference among patients with hyperkalemia: HK costs

Matched comparisons without hyperkalemia

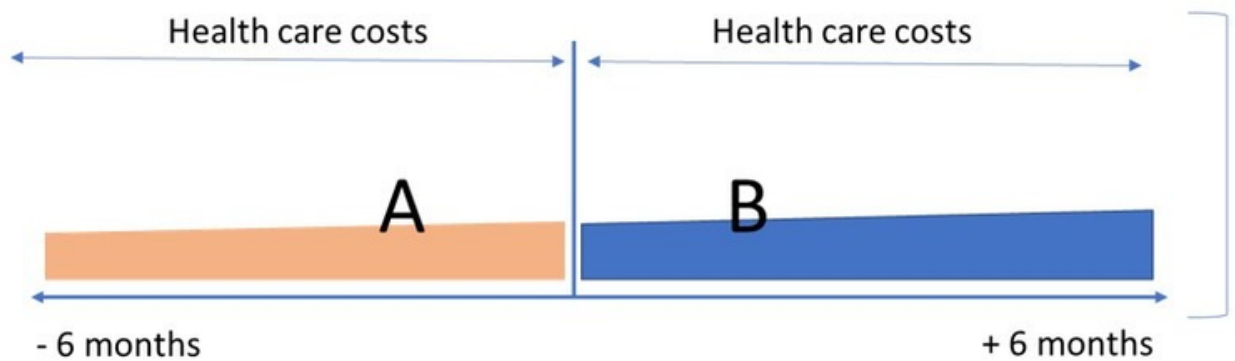

Costs $B-\operatorname{costs} A=$ Cost difference related to natural disease course among nonhyperkalemia patients: non-HK costs

The costs associated with hyperkalemia $=\mathrm{HK}$ costs - non- $\mathrm{HK}$ costs

\section{Matching criteria for hyperkalemia patients and comparison patients without hyperkalemia}

\begin{tabular}{|l|l|}
\hline Patient cohort & Matching criteria \\
\hline Chronic kidney disease & $\begin{array}{l}\text { Age, gender, Charlson Comorbidity Index score, chronic kidney disease duration, } \\
\text { calendar year of CKD diagnosis, CKD stage based on eGFR level, presence of heart } \\
\text { failure, diabetes or hypertension, hyperkalemia-associated drugs }{ }^{1}\end{array}$ \\
\hline Age, gender, Charlson Comorbidity Index score, heart failure duration, calendar \\
\hline yeart failure & hypertension, eGFR level, hyperkalemia-associated drugs ${ }^{1}$ \\
\hline Age, gender, Charlson Comorbidity Index score, diabetes duration, calendar year of \\
diabetes diagnosis, micro- or macrovascular complications, HbA1c level
\end{tabular}

${ }^{1} \mathrm{ACEis} / \mathrm{ARBs}$, spironolactone or eplerenone, or potassium supplements at the time of hyperkalemia

Figure 1 Overall study design and matching factors.

her costs from 1 April 2008 to 31 September 2008, minus his or her costs from 1 October 2007 to 31 March 2008 (figure 1).

To control for any changes in HRU and costs related to the natural disease course over time, such as CKD or HF progression, we selected a group of matched comparisons without HK within each disease cohort. Thus, for each patient who developed HK, we selected, by nearest neighbour matching, one comparison patient among those who were alive and had not yet developed HK on the index date of the corresponding matched HK patient, and who was as similar as possible for a range of matching factors. These factors are shown in figure 1 and included gender, patient age (in years), calendar year of first record of the disease, disease duration (in days), Charlson Comorbidity Index score $(0,1,2,3+)$, as well as additional prespecified 
clinical factors specific to each of the three disease cohorts (figure 1).

The total costs associated with HK were then estimated as the cost difference 6 months before and after the HK event among the HK patients minus the cost difference during the same period among the non-HK comparisons (figure 1). Hence, HK-associated costs were derived as a difference-in-difference, in which the difference between costs for HK patients and non-HK comparisons were regarded to be associated with the HK event (figure 1). ${ }^{27}$

To account for early mortality during the 6 months following the index date in both the HK patients and non-HK comparisons, an additional analysis of mean cost per risk time was performed, where costs were weighted by time-at-risk within the 6 months. We also conducted a supplementary analysis in which the difference-in-difference costs were restricted to costs 1 month before the HK event and 1 month after the HK event. The CIs in the main analysis were calculated based on the assumption that the difference-in-difference followed a t-distribution. The CIs in the subanalysis where the costs were weighted by time-atrisk were calculated based on bootstrapping.

\section{Unit costs}

Costs for hospital admissions and outpatient contacts (including emergency room visits) were based on the Diagnosis-Related Groups (DRG) and Danish Outpatient Grouping System charges. ${ }^{28}$ Costs of GP consultations and contacts such as phone calls, tests and mileage allowance were calculated based on prescheduled fees for GP services in the Danish healthcare system. ${ }^{24}$ Drug acquisition costs for drugs prescribed on an outpatient basis were calculated based on Danish pharmacy retail prices. ${ }^{29}$ All unit costs were based on the calendar year in which the resource utilisation occurred and converted to the year 2018 costs, using the official healthcare sector price index published by Statistics Denmark. ${ }^{30}$ All costs were converted to Euros using an average 2018 exchange rate, according to the European Central Bank, of 7.45 DKK per Euro.

\section{RESULTS}

Among 78372 patients with CKD, 14233 patients with HF and 37479 patients with diabetes, one or more HK events were experienced by 17747 (23\%) of the CKD patients; 5141 (36\%) of HF patients; and 4183 (11\%) of the diabetes patients. Among the 27071 patients with HK, those with HF were older (median age was 79 vs 76 in CKD patients and 69 in diabetes patients), included more patients with eGFR levels $<30 \mathrm{~mL} / \mathrm{min} / 1.73 \mathrm{~m}^{2}(42 \%$ vs $37 \%$ in CKD patients and 24\% in diabetes patients), and had a higher proportion of ACE inhibitor users (55\%) than the CKD (43\%) or diabetes patients (48\%) (table 1). Similar differences by disease group were observed for the 26900 comparison patients without HK (online supplementary table $\mathrm{S} 1$ ).

\section{HK incidence}

The incidence rates of HK were 99.0, 256.7 and 45.7 per 1000 person-years among the CKD, HF and diabetes patients, respectively (figures 2-4). The incidence of more severe HK events, $>5.5$ and $>6.0 \mathrm{mmol} / \mathrm{L}$, was lower across the three disease cohorts. Among the HF patients, more patients had more severe HK $(>5.5 \mathrm{mmol} / \mathrm{L})(18 \%)$, compared with the CKD patients $(10 \%)$ and diabetes patients (4\%). The baseline characteristics of the patients with more severe HK events $(>5.5$ and $>6.0 \mathrm{mmol} / \mathrm{L}$ ) and of their matched comparisons without HK are reported in online supplementary tables S2 and S3. A large proportion of the patients with a first HK event experienced a second HK event; $44 \%$ of the CKD patients, $44 \%$ of the HF patients and $45 \%$ of the diabetes patients. Among these surviving patients, an increasing proportion suffered subsequent HK events $(>5.0 \mathrm{mmol} / \mathrm{L})$, and the time between HK events was successively shorter for the subsequent events (figures 2-4). After an initial HK event $(>5.0 \mathrm{mmol} / \mathrm{L})$, subsequent $\mathrm{HK}$ events were more frequently detected in primary care than in hospitals, whereas for patients with severe HK events $(>6.0 \mathrm{mmol} / \mathrm{L})$, subsequent HK events were predominantly diagnosed in the hospital setting (figures 2-4).

\section{HRU and costs associated with HK}

Among the CKD patients with a HK event, mean numbers of acute hospital admissions increased from 0.8 during the period of 6 months before HK to 1.2 during the period of 6 months after HK (table 2). Among the HF patients with a HK event, corresponding acute admissions increased from 1.3 to 1.5 and among the diabetes patients with a HK event from 0.7 to 1.0. One-third (diabetes) to one-half (HF) of the HK patients experienced an acute admission at the time of their HK event; ICD-10 chapters for primary discharge diagnoses for these admissions are shown in online supplementary table S4. Mean costs of acute admissions with ventilator treatment and ICU stay were much higher after versus before HK, by 5.2-fold and 4.6-fold, respectively, in CKD patients, 4.6-fold and 3.7-fold in HF patients and 8.5-fold and 6.0-fold in diabetes patients (table 2). Among the matched non-HK comparisons in the same period, minor differences in mean numbers of non-acute hospitalisations, and outpatient and GP visits were observed. The mean difference per patient in total costs among HK patients between 6 months before and 6 months after HK was $€ 5518$ in CKD patients, $€ 5141$ in HF patients and $€ 4650$ in diabetes patients (table 2, figure 5). In comparison, the mean difference in total costs among the matched non-HK patients between 6 months before and 6 months after the index date was $€ 441$ in CKD patients, $€-887$ in HF patients and $€-212$ in diabetes patients, resulting in estimated HK-associated costs of $€ 5076$ (95\% CI 4690 to 5463) in patients with CKD, $€ 6018$ (95\% CI 5234 to 6802) in patients with $\mathrm{HF}$ and $€ 4862$ (95\% CI 4156 to 5568) in patients with diabetes. Overall, costs after HK increased by $71 \%$ in CKD patients, $52 \%$ in $\mathrm{HF}$ patients and $70 \%$ in diabetes patients. 
Table 1 Baseline characteristics of patients with hyperkalaemia $(>5.0 \mathrm{mmol} / \mathrm{L})$

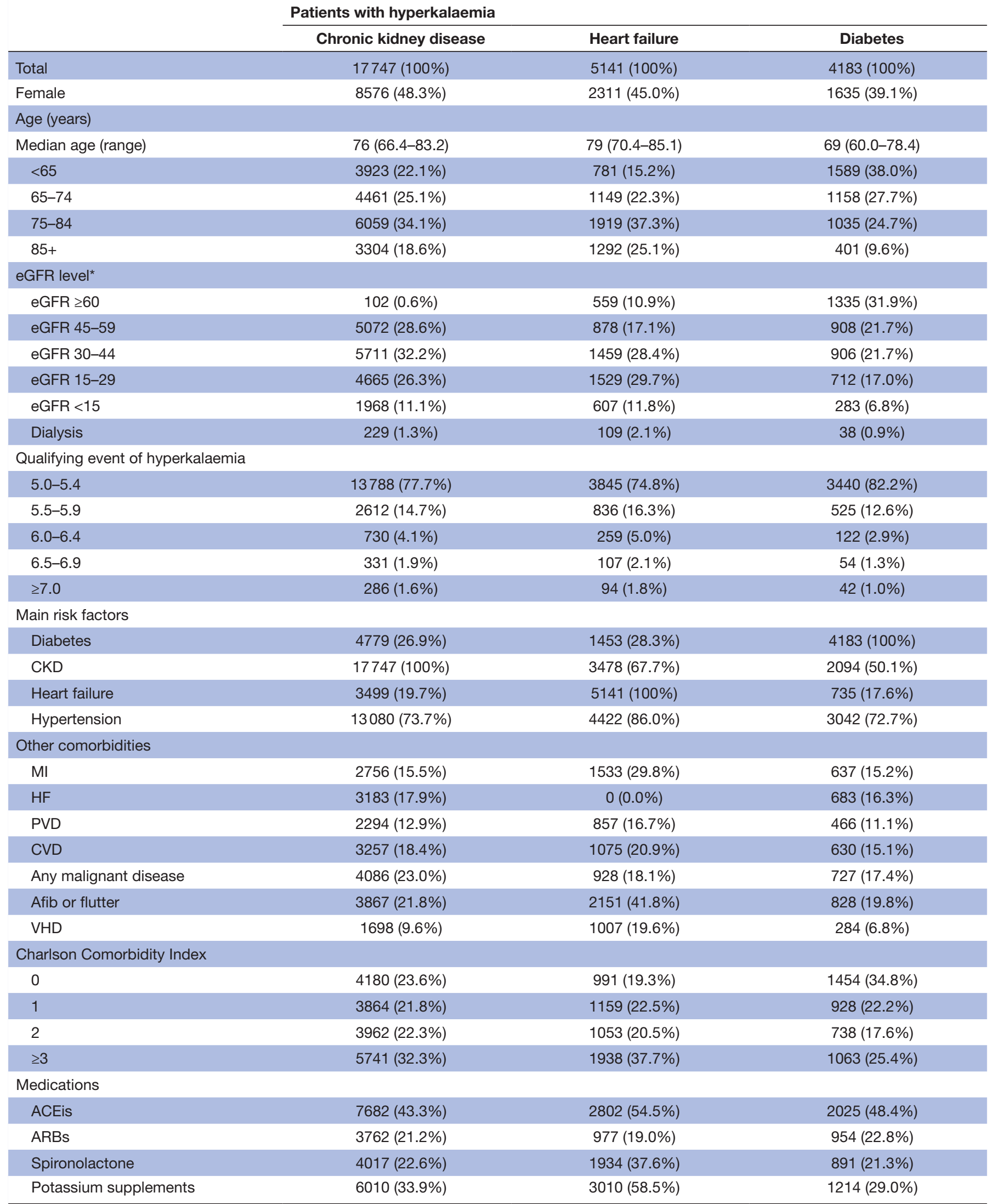

${ }^{*} \mathrm{~mL} / \mathrm{min} / 1.73 \mathrm{~m}^{2}$.

ACEis, angiotensin converting enzyme inhibitors; ARBs, agiotensin II receptor blockers; CVD, cardiovascular disease; HF, heart failure; MI, myocardial infarction; PVD, peripheral vascular disease; VHD, valvular heart disease. 


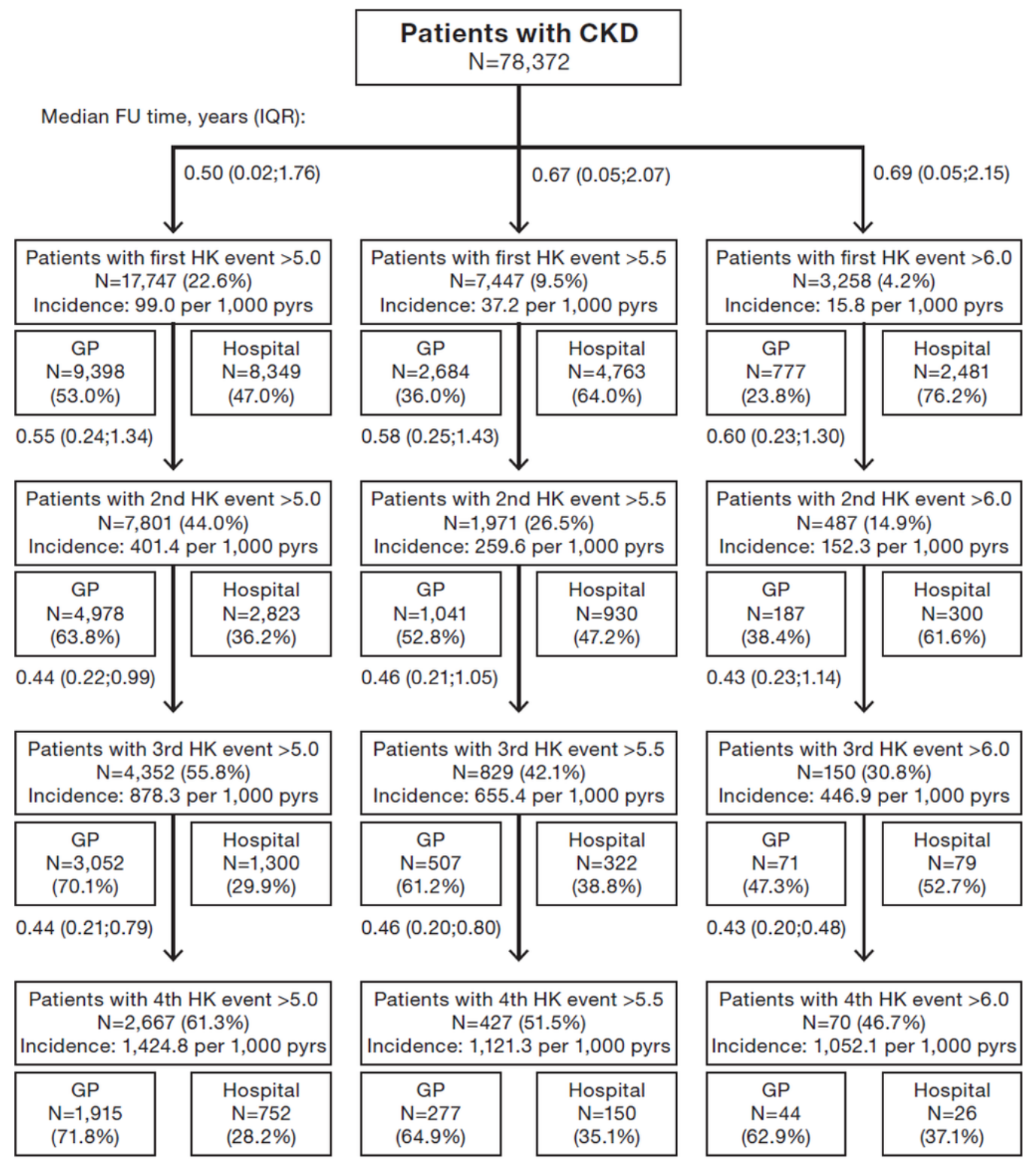

${ }^{*}$ CKD: Chronic kidney disease, FU: Follow-up, IQR: Interquartile Range, HK: Hyperkalemia, GP: General practitioner.

Figure 2 Risks of developing first and subsequent events with elevated potassium level $>5.0,>5.5$ and $>6.0 \mathrm{mmol} / \mathrm{L}$ in patients with chronic kidney disease and split between diagnosis setting of hyperkalaemia.

The HK-associated costs were higher in patients with more severe HK events $(>5.5$ and $>6.0 \mathrm{mmol} / \mathrm{L}$ ) (online supplementary tables S5 and S6). The higher costs after versus before incident $\mathrm{HK}$ and the cost amplification by HK severity are shown in table 2 and depicted in figure 5 .

Considering the high mortality 6 months after the HK event among the HK patients compared with the matched non-HK comparisons, $(27 \%$ vs $7 \%$ mortality in CKD patients, $35 \%$ vs $15 \%$ mortality in HF patients and $19 \%$ vs $4 \%$ mortality in diabetes patients, data not shown), the HK-associated costs within 6 months weighted by timeat-risk were estimated; $€ 8291$ (95\% CI 7823 to 8704 ) in CKD patients, $€ 11078$ (95\% CI 10031 to 12 034) in HF patients and $€ 6719$ (95\% CI 5943 to 7440 ) in diabetes patients (online supplementary table S7).

When restricting the calculations to costs 1 month before the HK event and 1 month after the HK event,
HK-associated costs were $€ 4017$ (95\% CI 3785 to 4249 ) in CKD patients, $€ 5140$ (95\% CI 4672 to 5607 ) in HF patients and $€ 3678$ (95\% CI 3280 to 4076 ) in diabetes patients (online supplementary table S8).

\section{DISCUSSION}

This population-based cohort study provides an overview of incidences of HK event and the corresponding HRU and costs, in patients with CKD, $\mathrm{HF}$ and diabetes during a maximum observation period of 5.5 years in Denmark. Overall, 17747 CKD patients, $5141 \mathrm{HF}$ patients and 4183 diabetes patients with a first HK event were identified. More than $40 \%$ of the patients had subsequent HK events, with successively shorter time between the events.

In CKD patients, the overall mean costs were $€ 5518$ higher after HK event compared with prior HK event 


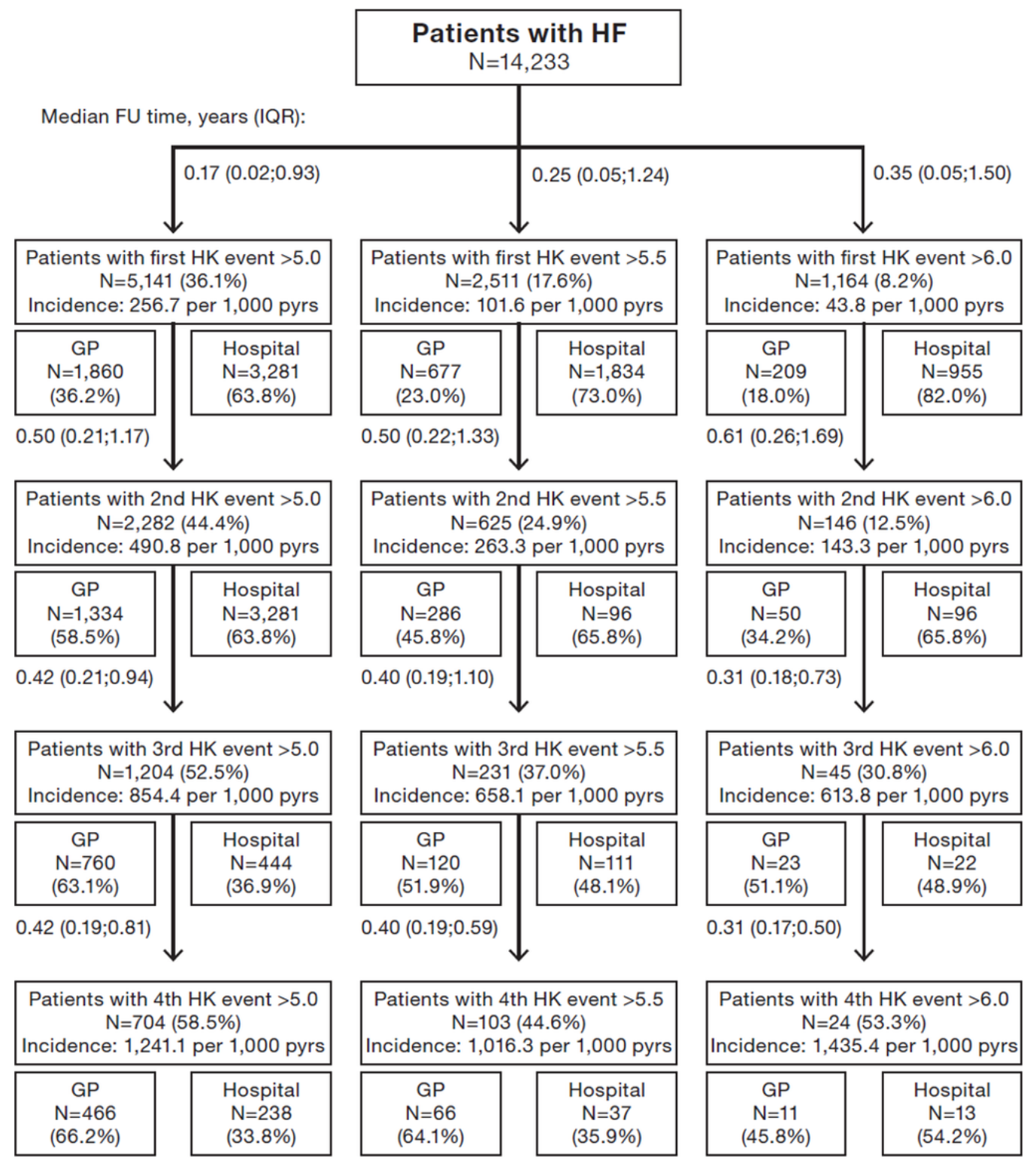

*HF: Heart failure, FU: Follow-up, IQR: Interquartile Range, HK: Hyperkalemia, GP: General practitioner.

Figure 3 Risks of developing first and subsequent events with elevated potassium level $>5.0,>5.5$ and $>6.0 \mathrm{mmol} / \mathrm{L}$ in patients with heart failure and split between diagnosis setting of hyperkalaemia.

while the costs in the matched non-HK comparisons was $€ 441$ during the same time period, resulting in a HK-associated cost of $€ 5076$. Corresponding mean costs associated with a first HK event were $€ 6018$ in patients with HF, and $€ 4862$ in patients with diabetes.

The difference in cost incurred by HK patients and non-HK patients was mainly driven by acute hospitalisations, where ICU stays and ventilator treatments were the main contributing components. The mean cost per acute hospitalisation, that is, due to longer hospital stays and/or a hospital episode with complications, was higher among HK patients, which may indicate hospitalisation for more severe conditions among the HK patients. Costs for primary care visits and prescribed drugs had a minor impact on the overall cost pattern associated with a HK event.
A larger proportion of HF patients had experienced mild and severe HK events, which occurred closer to the date of HF diagnosis, compared with the corresponding results in the CKD and diabetes patients. Relatively more HK events were detected in the hospital than in the primary care setting among the HF patients. The HF cohort also had a higher mean cost per patient prior to the HK event than had the CKD and diabetes cohort, and a larger absolute increase in mean costs after the HK event compared with the other disease cohort. The mean relative increase in costs associated with $\mathrm{HK}$, was higher among the CKD $(71 \%)$ and diabetes patients (70\%) compared with the HF patients $(52 \%)$. However, when taking the higher mortality among the HF patient cohort into account (35\% were deceased within 6 months after HF diagnosis), by weighing in patient survival time, 


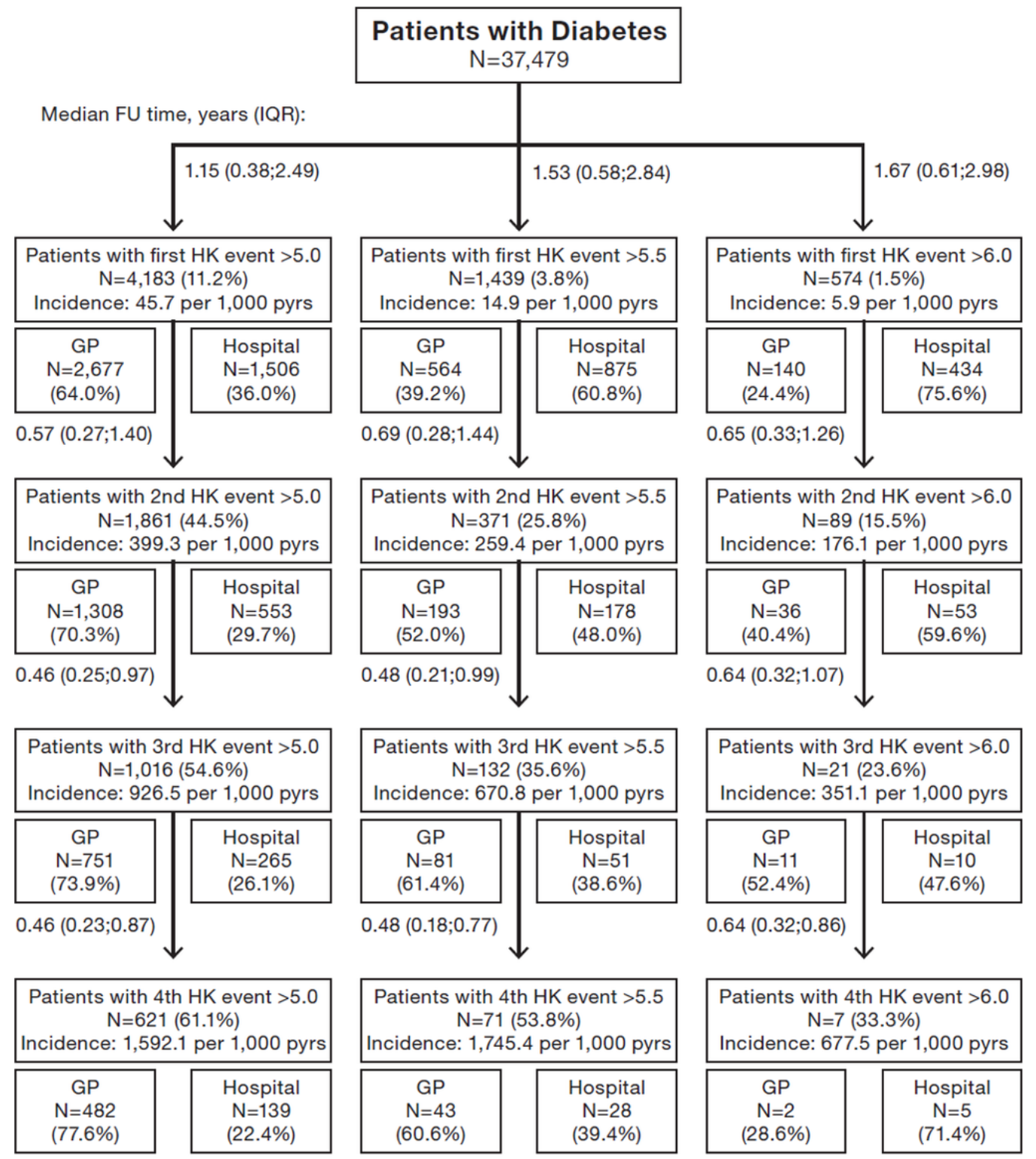

*FU: Follow-up, IQR: Interquartile Range, HK: Hyperkalemia, GP: General practitioner.

Figure 4 Risks of developing first and subsequent events with elevated potassium level $>5.0,>5.5$ and $>6.0 \mathrm{mmol} / \mathrm{L}$ in patients with diabetes and split between diagnosis setting of hyperkalaemia.

a higher relative mean cost increase was still observed for the HF patients compared with the other disease cohort.

For more severe HK episodes $(>5.5$ and $>6.0 \mathrm{mmol} / \mathrm{L}$, respectively), a higher mean cost was observed among the CKD and diabetes patients, compared with costs associated with milder HK events. This pattern was not observed among the HF patients, for whom the HK associated with cost did not differ greatly by the increasing severity of HK events. This finding might be partly explained by the particularly high early mortality among HF patients with severe HK.

Following the initial HK event, a larger proportion of the subsequent HK events were detected in a primary care compared with hospital setting. Following an initial HK event, patients would presumably undergo more frequent blood testing in primary care, thus potentially explaining why a larger proportion of the subsequent HK events were detected in primary care.

The few existing studies investigating HK-related costs in the literature, are mostly from the USA. Fitch et al estimated that monthly severity-adjusted CKD costs for HK patients were $\$ 4922$ versus $\$ 2036$ for those without HK. ${ }^{31}$ Castro and Coresh estimated a monthly cost of $\$ 5994$ and an annual cost of $\$ 31884$, to manage CKD patients who experienced HK, but without reference to a comparison group. ${ }^{32}$ Probably related to the considerable differences in clinical practice and in reimbursement system between the US and European/Nordichealthcare systems, the mean estimates and the magnitude of cost differences between HK and non-HK patients were generally higher in the US studies than in the current study, that is, our HK-associated costs converted to US $\$$ were $\$ 5837$ in the 


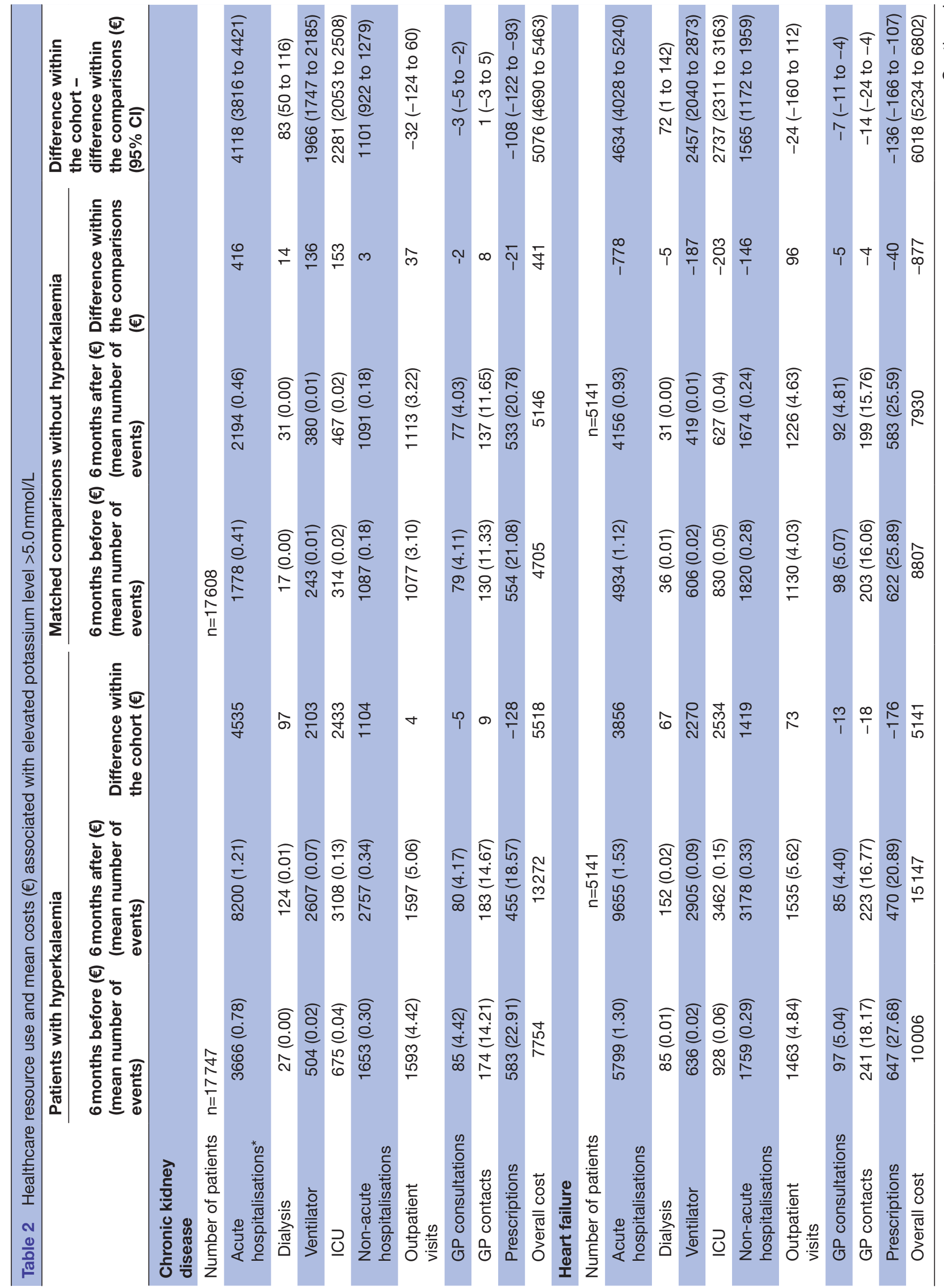

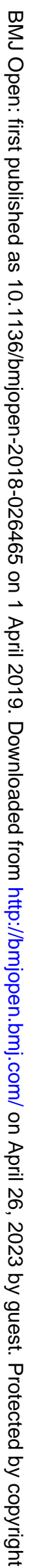




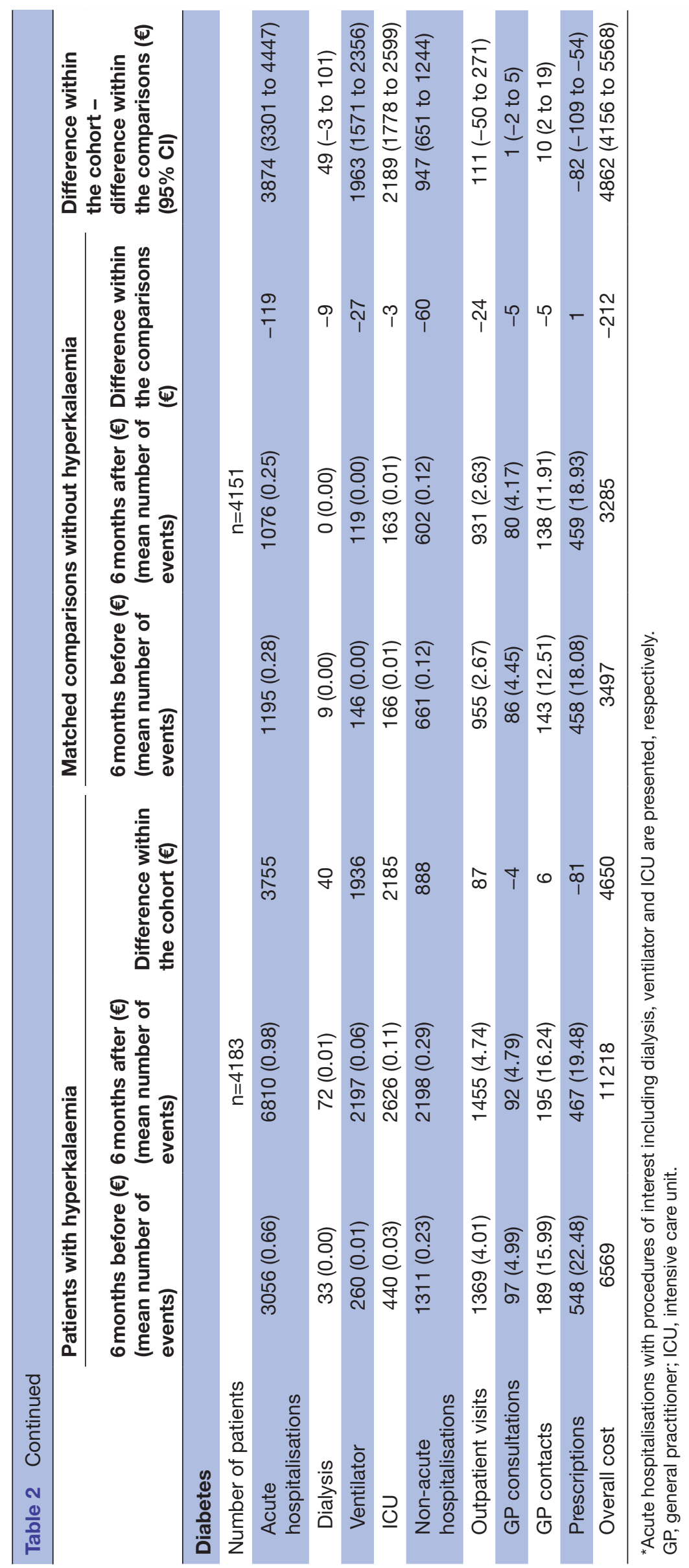


Patients with chronic kidney disease

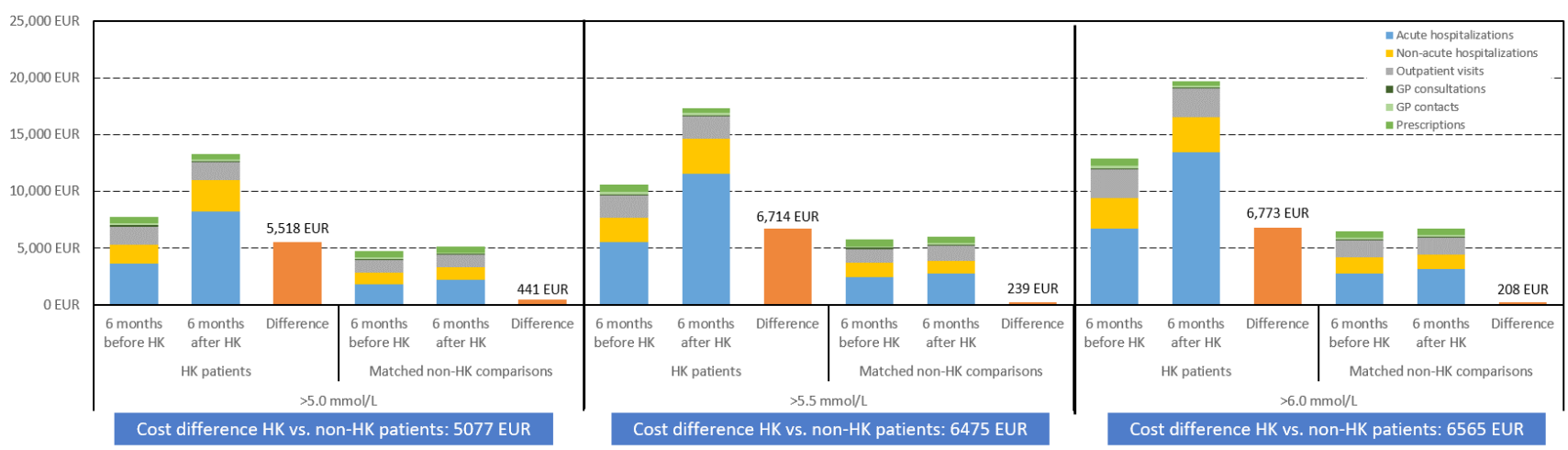

Patients with heart failure

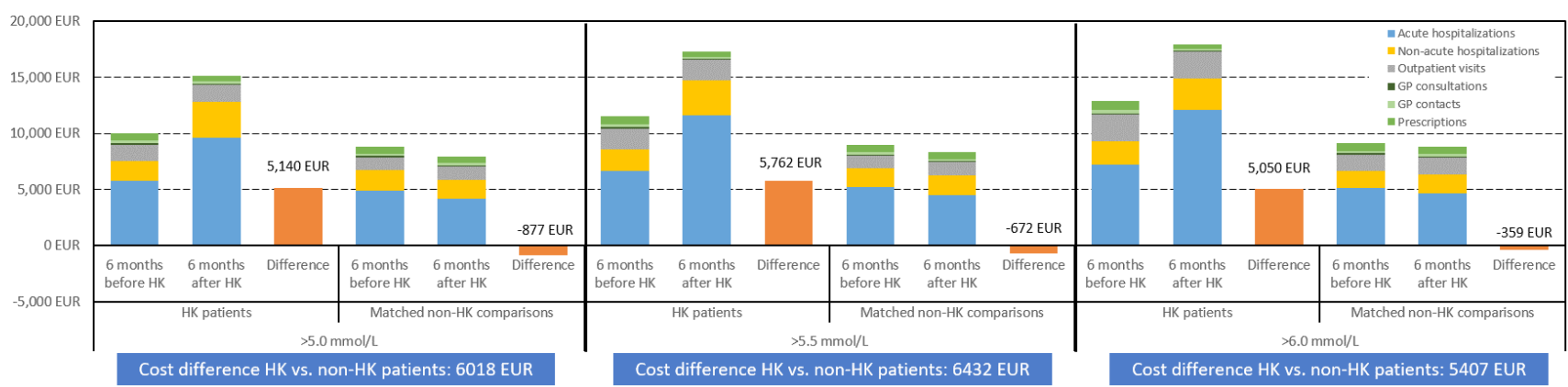

Patients with diabetes

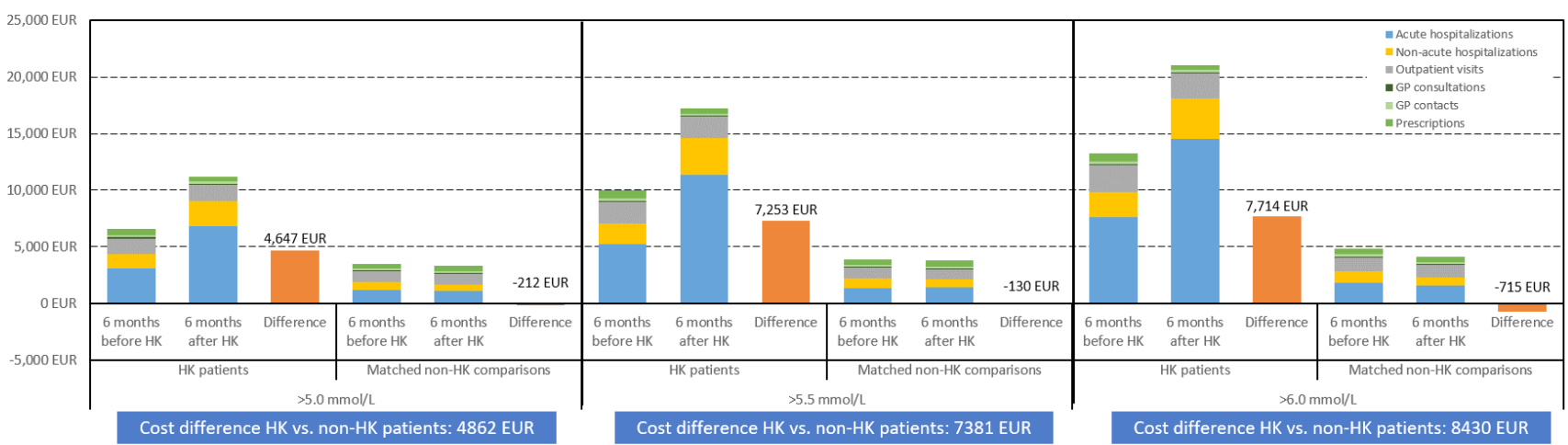

Figure 5 Mean cost of healthcare utilisation 6 months before and 6 months after HK, in chronic kidney disease, heart failure and diabetes patients, HK patients versus matched non-HK comparisons. GP, general practitioner; HK, hyperkalaemia.

CKD patients, \$6921 in the HF patients and \$5591 in the diabetes patients (applying an exchange rate of $€ 1$ $=\mathrm{US} \$ 1.15$ ).

This study does not come without limitations. First, any conclusions concerning causal mechanisms underlying HK outcomes and corresponding costs should be made with caution. Rather than precipitating a hospitalisation, elevated potassium levels measured during a hospitalisation may stem from an underlying condition that led to hospitalisation (eg, infection, dehydration, deteriorating kidney function) among the disease cohorts examined. ${ }^{25}{ }^{26}$ We observed a variety of acute disease diagnoses, typically for an elderly highly comorbid patient population. On the other hand, HK itself may have had a bearing on the acute hospitalisation and its severity and course, and HK may also have directly led to admissions, for example, through muscle weakness, cardiac problems, resulting falls, etc. The exact order of events in the pathophysiological pathway leading to a hospitalisation is difficult to disentangle, especially for elderly comorbid patients. Therefore, it is not yet predictable how the effective and sustained management of HK will affect the corresponding costs associated with HK occurrence.

Second, our study is reliant on routine care ICD-10 codes for morbidity data and therefore, the possibility of coding errors cannot be ruled out. Furthermore, we did not have access to detailed clinical data for, for example, severity of HF, such as ejection fraction or New York Heart Association Functional Classification, or for exact type of 
diabetes, which remains uncertain based on treatment modality and hospital codes alone.

Third, inclusion of patients in the study cohorts was restricted to 2005-2011 due to DRG record availability and feasibility of long-term follow-up. Clinical management of the comorbid conditions that are known risk factors for HK, as well as HK management, may have changed during this period; however, pharmacological advancements in HK management have been relatively stagnant within this time period..$^{33} 34$

Fourth, long-term clinical implications of HK and the corresponding HRU and costs were not investigated. This could, however, be a field for further work and investigation.

Lastly, information on drugs administered during hospitalisations, including those for the management of HK, were not available in the dataset. Therefore, their acquisition costs could not be examined in detail, but only included as a part of the cost of each DRG hospital episode.

\section{CONCLUSIONS}

The costs associated with incident HK were substantial among CKD, $\mathrm{HF}$ and diabetes patients, and were mainly driven by increased use of hospital-based care. A large proportion of patients experienced subsequent HK events after a first incident event, with a successively shorter time between events. Our findings indicate that the high HRU and corresponding costs associated with HK events, as well as the recurring pattern of events among CKD, HF and diabetes patients, constitute a substantial clinical and economic burden for patients, healthcare providers and payers. With our observational study design, it was not possible to determine whether HK was the direct cause of hospital admissions and increased HRU. However, since HK is at least partly contributing to the substantial adverse health outcomes observed, timely detection and management of HK among high-risk populations and avoidance of subsequent events may translate not only to clinical benefits for the patients but may also alleviate the economic burden for healthcare providers and payers. Additional research on the long-term costs, particularly for patients with recurrent events of HK, will be useful to inform clinical decision-making.

Acknowledgements The authors would like to thank Gunilla Telg and Susan Grandy (AstraZeneca employees) for their support throughout the conduct of this study.

Contributors Conception and design: EP, KK, RWT and SKN. Analysis and interpretation of data: SKN, KK, RWT, EP, LPH and HTS. Drafting the article: KK, EP, RWT and LPH. Revising the article: KK, RWT, LPH, EP, HTS and SKN. Providing intellectual content of critical importance to the work described: all authors. Final approval of the version to be published: all authors.

Funding This work was supported by a research grant from AstraZeneca to Aarhus University and by the Program for Clinical Research Infrastructure (PROCRIN) established by the Lundbeck Foundation and the Novo Nordisk Foundation. Employees of AstraZeneca contributed to study design, analysis and interpretation of the data, as well as drafting of this manuscript.
Competing interests KK, LPH and EP are employees of AstraZeneca. RWT, SKN and HTS have reported no personal conflicts of interest relevant to this article. The Department of Clinical Epidemiology is, however, involved in studies with funding from various companies as research grants to (and administered by) Aarhus University, including the present study.

Patient consent for publication Not required.

Ethics approval The ethics approval to utilise patient records from the data sources was granted by an institutional review board in Aarhus University Hospital.

Provenance and peer review Not commissioned; externally peer reviewed.

Data sharing statement The datasets used and/or analysed during the current study cannot be shared according to Danish legislation; source data from the Danish registries can be applied for at the Danish National Health Data Authority(Sundhedsdatastyrelsen).

Open access This is an open access article distributed in accordance with the Creative Commons Attribution Non Commercial (CC BY-NC 4.0) license, which permits others to distribute, remix, adapt, build upon this work non-commercially, and license their derivative works on different terms, provided the original work is properly cited, appropriate credit is given, any changes made indicated, and the use is non-commercial. See: http://creativecommons.org/licenses/by-nc/4.0/.

\section{REFERENCES}

1. Kidney Disease Outcomes Quality Initiative (K/DOQI). K/DOQI clinical practice guidelines on hypertension and antihypertensive agents in chronic kidney disease. Am J Kidney Dis 2004;43(5 Suppl 1):S1-290.

2. Treatment of hyperkalemia in Denmark. http://pro.medicin.dk/ Sygdomme/Sygdom/318155 (cited 07 Jan 2019).

3. Fleet JL, Shariff SZ, Gandhi S, et al. Validity of the International Classification of Diseases 10th revision code for hyperkalaemia in elderly patients at presentation to an emergency department and at hospital admission. BMJ Open 2012;2:e001821.

4. Drawz PE, Babineau DC, Rahman M. Metabolic complications in elderly adults with chronic kidney disease. J Am Geriatr Soc 2012;60:310-5.

5. Martin Perez MM, Michel A, Garcia Rodriguez LA. Incidence of hyperkalemia in patients with newly diagnosed heart failure: A large observational study in the UK. European Journal of Heart Failure Conference, Seville, Spain, 2015:426

6. Ahmed J, Weisberg LS. Hyperkalemia in dialysis patients. Semin Dial 2001;14:348-56.

7. Jain N, Kotla S, Little BB, et al. Predictors of hyperkalemia and death in patients with cardiac and renal disease. Am J Cardiol 2012;109:1510-3.

8. Hayes J, Kalantar-Zadeh K, Lu JL, et al. Association of hypo- and hyperkalemia with disease progression and mortality in males with chronic kidney disease: the role of race. Nephron Clin Pract 2012;120:c8-c16.

9. Bandak G, Sang Y, Gasparini A, et al. Hyperkalemia After Initiating Renin-Angiotensin System Blockade: The Stockholm Creatinine Measurements (SCREAM) Project. J Am Heart Assoc 2017;6.

10. An JN, Lee JP, Jeon HJ, et al. Severe hyperkalemia requiring hospitalization: predictors of mortality. Crit Care 2012;16:R225.

11. Conway R, Creagh D, Byrne DG, et al. Serum potassium levels as an outcome determinant in acute medical admissions. Clin Med 2015;15:239-43.

12. Park KS, Kim JH, Ku EJ, et al. Clinical risk factors of postoperative hyperkalemia after adrenalectomy in patients with aldosteroneproducing adenoma. Eur J Endocrinol 2015;172:725-31.

13. Korgaonkar S, Tilea A, Gillespie BW, et al. Serum potassium and outcomes in CKD: insights from the RRI-CKD cohort study. Clin J Am Soc Nephrol 2010;5:762-9.

14. Luo J, Brunelli SM, Jensen DE, et al. Association between serum potassium and outcomes in patients with reduced kidney function. Clin J Am Soc Nephrol 2016;11:90-100.

15. Thomsen RW, Nicolaisen SK, Hasvold P, et al. Elevated potassium levels in patients with chronic kidney disease: occurrence, risk factors and clinical outcomes-a Danish population-based cohort study. Nephrol Dial Transplant 2018;33:1610-20.

16. Aldahl M, Jensen AC, Davidsen L, et al. Associations of serum potassium levels with mortality in chronic heart failure patients. Eur Heart J 2017;38:2890-6.

17. Dunn JD, Benton WW, Orozco-Torrentera E, et al. The burden of hyperkalemia in patients with cardiovascular and renal disease. $\mathrm{Am} J$ Manag Care 2015;21(15 Suppl):s307-15. 
18. Smith DH, Raebel MA, Chan KA, et al. An economic evaluation of a laboratory monitoring program for renin-angiotensin system agents. Med Decis Making 2011;31:315-24.

19. Chazard E, Dumesnil C, Beuscart R. How much does hyperkalemia lengthen inpatient stays? About methodological issues in analyzing time-dependant events. Stud Health Technol Inform 2015;210:835-9.

20. Denmark S. Population and population projections by Statbank Denmark. (cited 07 Jan 2019).

21. Grann AF, Erichsen R, Nielsen AG, et al. Existing data sources for clinical epidemiology: The clinical laboratory information system (LABKA) research database at Aarhus University, Denmark. Clin Epidemiol 2011;3:133-8.

22. Andersen JS, Olivarius NF, Krasnik A. The Danish National Health Service Register. Scand J Public Health 2011;39(7 Suppl):34-7.

23. Pottegård A, Schmidt SAJ, Wallach-Kildemoes H, et al. Data Resource Profile: The Danish National Prescription Registry. Int J Epidemiol 2017;46:798-f.

24. Praktiserende Lægers Organisation. Honorarer og takster. https:// www.laeger.dk./english (cited 07 Jan 2019).

25. Thomsen RW, Nicolaisen SK, Hasvold P, et al. Elevated Potassium Levels in Patients With Congestive Heart Failure: Occurrence, Risk Factors, and Clinical Outcomes: A Danish Population-Based Cohort Study. J Am Heart Assoc 2018;7.

26. Thomsen RW, Nicolaisen SK, Adelborg K, et al. Hyperkalaemia in people with diabetes: occurrence, risk factors and outcomes in a Danish population-based cohort study. Diabet Med 2018;35:1051-60.

27. Jakobsen M, Kolodziejczyk C, Klausen Fredslund E, et al. Costs of major intracranial, gastrointestinal and other bleeding events in patients with atrial fibrillation - a nationwide cohort study. BMC Health Serv Res 2017;17:398.

28. SUNDHEDSDATASTYRELSEN. DRG-takster. $2018 \mathrm{https} / / /$ sundheds datastyrelsen.dk/da/afregning-og-finansiering/takster-drg/takster2018 (cited 2019 Jan 07).

29. Danish Medicines Agency. Pharmaceutical price database. https:// laegemiddelstyrelsen.dk/en/ (cited 07 Jan 2019).

30. Official healthcare sector price index. https://www.dst.dk/en (cited 08 Jan 2019).

31. Fitch KW, Engel J, Blumen $\mathrm{H}$. An Evaluation of the Burden of Hyperkalemia in the Medicare Population. Journal of Managed Care \& Specialty Pharmacy 2016;22.

32. Castro AF, Coresh J. CKD surveillance using laboratory data from the population-based National Health and Nutrition Examination Survey (NHANES). Am J Kidney Dis 2009;53(3 Suppl 3):S46-55.

33. Zannad F, Rossignol P, Stough WG, et al. New approaches to hyperkalemia in patients with indications for renin angiotensin aldosterone inhibitors: Considerations for trial design and regulatory approval. Int J Cardiol 2016;216:46-51.

34. Epstein M, Pitt B. Recent advances in pharmacological treatments of hyperkalemia: focus on patiromer. Expert Opin Pharmacother 2016;17:1435-48. 